\title{
Monitoring the Radioactive Contamination of Some Surfaces
}

\author{
Anas Shehu ${ }^{1 *}$, Iliyasu Usman ${ }^{2}$, Ahmad Rufa'i ${ }^{3}$, Jamilu A. Kwari ${ }^{4}$ \\ ${ }^{1,2}$ Department of Physics, Sokoto State University, Sokoto, Nigeria ${ }^{1}$ \\ ${ }^{3}$ Department of Mathematics, Sokoto State University, Sokoto Nigeria \\ ${ }^{4}$ Department of Physics, College of Agriculture and Animal Science Wurno, Sokoto, Nigeria
}

\begin{abstract}
This paper describes the typical process for monitoring surfaces contaminated by different sources, such as strontium $\left({ }^{90} \mathrm{Sr}\right)$, cobalt $\left({ }^{60} \mathrm{Co}\right)$ and chlorine $\left({ }^{36} \mathrm{Cl}\right)$, in a working environment. There are several detectors that are used to identify both the location and activity of a contamination source. In this report, the most effective detector to scan and monitor Beta-rays is the mini-monitor $\mathrm{E}$, with an efficiency of $\mathbf{4 2 . 3 \%}$, $20 \%$ and $14 \%$ for detecting ${ }^{90} \mathrm{Sr},{ }^{36} \mathrm{Cl}$ and ${ }^{60} \mathrm{Co}$, respectively. However, the NE BP/4A/4C scintillator detector was the most suitable for detecting Gamma-rays. In addition, a smear test was carried out by using a high-efficiency G-M tube and several different sources of ${ }^{90} \mathrm{Sr}$. The decontamination process successfully reduced the contamination level to almost $2.1 \%$ of the original percentage of contamination when the first wipe was done.
\end{abstract}

Keywords : Strontium, Cobalt, Chlorine, Detectors etc

\section{INTRODUCTION}

$\mathrm{T}$ surfaces are occasionally contaminated by radioactivity either accidently or due to a misuse of radioactive resources. Generally, anything in the radiation laboratory can be exposed to the contamination, such as the air, water, apparatus and clothing. The people working in the laboratory or nuclear industries can also be exposed to the contamination, but this is generally just external; e.g., the radioactive material sticks to the person's clothes or they touch a contaminated surface. Internal contamination can happen if a person drinks contaminated water or inhales contaminated air, but this is less common. Statistically, surface contamination limits are usually determined depending on the type of contaminant. For instance, the limits of Beta, Gamma emitters and low-toxicity Alpha emitters are calculated as $4 \mathrm{Bqcm}^{2}$, while all other alpha-emitting radionuclides are about $0.4 \mathrm{Bqcm}^{2}$ (Canada et al., 2008). This report discusses the best methods for determining the activity level on a contaminated surface using different types of detectors. The measured activity levels of various contaminants and assessed the ability of a few detectors to identify the different radioactive materials with different energy levels and then decontaminated the contaminated area using an appropriate solution.

\section{Theory}

There are several ways to detect radioactive material, most of which depend on the ionization process that happens when gamma-rays or charge particles interact with the gas, liquid, or solid form of the detector to produce ion pairs (Baron, Kulkarni, and Willeke, 2011 ). Even though a G-M monitor usually gives results as a count rate, it can be converted to activity $\mathrm{Bqcm}^{2}$ using the calibration factor (Rahman, 2008).

To calibrate the monitor, the formula below can be used

$C F=R C / S C$

$R C$ is the surface emission and $\mathbf{S C}$ is the observed count rate.

$C F=\left((N / A S) X\left(D A /{ }_{\text {CRo }}\right)\right) X 1000$

$C F$ is the calibration factor

$N$ is the activity at the current time

$A S$ is the area of the source

$D A$ is the sensitive area of the detector

$C R_{o}$ is the average count rate background

Furthermore, the radioactive decay can be calculated by the radioactive decay formula,

$N=N_{0} e^{-\left(\frac{\operatorname{Ln} 2}{T_{1 / 2}}\right) t}$

$N$ is the activity at the current time

$N_{O}$ is the initial activity

$t$ is the time difference.

The precise reading was taken, and the monitor was held over the sensitive area of the source for approximately 2 to $4 \mathrm{sec}$, which is enough time for the monitor to count the rate properly. It would also be better if the distance between the probe and the source area is less than $1 \mathrm{~cm}$, especially with Beta and Gamma rays, as this increases the efficiency and accuracy of the monitor. If the probe becomes contaminated, this can confound the results, so it is important to avoid touching it to the surface (Rahman, 2008). Finally, to distinguish between Beta and Gamma emitters, a thin aluminium sheet can be placed over the sensitive area of the ${ }^{60} \mathrm{Co}$ source to block the low energy (Beta) and allow the Gamma rays to transmit. 
The efficiency level can be calculated using the following formula (Alvarez, Maia, and Caldas, 2005):

$E=\left(\frac{C-B}{A X S}\right) X 100$

$E$ is the instrument efficiency, the ratio between the instrument net reading and the surface emission rate of a source under same geometrical conditions;

$C$ is the measured total count rate $\left(\mathrm{s}^{-1}\right)$

$B$ is the background count rate $\left(\mathrm{s}^{-1}\right)$

$S$ is the sensitive window area of the measuring instrument $\left(\mathrm{cm}^{2}\right)$

$A$ is the surface emission rate per unit area of the reference source $\left(\mathrm{Bqcm}^{2}\right)$.

\section{METHOD}

The process of determining the radioactive contamination of a surface can be divided into two parts. The first part is monitor calibration and selection, while the second part is monitoring the surface contamination using Smear testing with proper decontamination and disposal procedures.

\section{Monitor calibration and selection}

Three different detectors were used, mini-monitor E (G-M tube), mini-monitor SL (G-M tube) and NE BP4/1 (scintillator), see Appendix 1 in the appendices. The minimonitors were calibrated using well known sources, namely

${ }^{90} \mathrm{Sr}$ (a Beta emitter), ${ }^{60} \mathrm{Co}$ (a Beta and Gamma emitter) and ${ }^{36} \mathrm{Cl}$ (Beta emitter). Furthermore, a thin aluminium sheet was used with ${ }^{60} \mathrm{Co}$ to block the low Beta energy. Moreover, the activity per unit area of each source was measured to calculate the calibration factor for each monitor; this was done by converting the results from count rate into actual source activity.

\section{Monitoring surface contamination}

We estimated the contamination level of a surface that had been contaminated with a Beta emitter phosphorus-32 $\left({ }^{32} \mathrm{P}\right)$ by dividing it into equal squares. It was then monitored with the detector chosen to evaluate the contamination stage.

\section{Smear testing with decontamination and disposal}

This section focused on the decontamination of the surface using a smear test. In this stage, a high-efficiency G-M tube was used to determine the level of activity of the surface after calibrating different known sources of ${ }^{90} \mathrm{Sr}$ which were used to produce the activity calibration curve. One wipe took in each region around the contaminated area to determine the activity levels which might influence on the activity of the contaminated area. Then, the decontamination process began with one wipe, followed by several wipes of the contaminated area. The wiping proper procedure was as follows: The filter papers were folded into a buttery shape and applied maximum pressure in a circular motion, moving from the outside to the inside of the contaminated area; and then put the wipe under the G-M tube to be measured (Protection against radiation from Brachytherapy sources, recommendations of the national council on radiation protection and measurements, 1972). Finally, the radioactive paper filters were disposed in a hazard-labelled glass container.

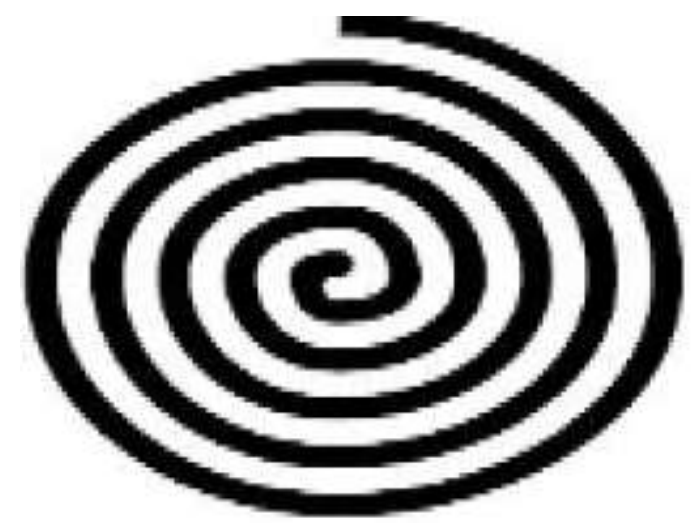

Figure 1: The way of wiping moving from outside to inside

\section{RESULTS AND DISCUSSION}

First, the activity of each source was calculated on the day of the experiment (26.10.2015) using the radioactive decay formula (Equation 3). The activity levels were $3.870 \mathrm{kBq}$, $1.211 \mathrm{kBq}$ and $2.700 \mathrm{kBq}$ for ${ }^{90} \mathrm{Sr},{ }^{36} \mathrm{Cl}$ and ${ }^{60} \mathrm{Co}$, respectively.

The next stage was calculating the calibration factors and efficiencies using (Equations 2 and 4). The results of the background count rates, count rates, background subtracted count rates, calibration factors and efficiencies are provided in detail in Appendix 2 to 5 in the appendices.

The bar chart in Figure 2 indicates that mini-monitor $\mathrm{E}$ was extremely efficient for detecting rays $(41 \%, 20 \%$ and $14 \%$ for ${ }^{90} \mathrm{Sr},{ }^{36} \mathrm{Cl}$ and ${ }^{60} \mathrm{Co}$ sources, respectively).

Conversely, the SL detector was the least efficient for detecting Beta rays, with just a mere $8 \%$ for ${ }^{90} \mathrm{Sr}$ and just under $1 \%$ for ${ }^{60} \mathrm{Co}$. It was also found that the efficiency proportions dropped sharply while using an aluminium sheet over ${ }^{60} \mathrm{Co}$ to block most of the Beta -rays. Nevertheless, NE BP4/4A was the best detector for detecting Gamma rays.

The next stage started with monitoring a plate that had been deliberately contaminated with ${ }^{32} \mathrm{P}$, which emits Beta-rays of 1.7 MeV over an area of $10 \mathrm{~cm}^{2}$.

Based on the data presented in Figure 2, the decision was made to use mini-monitor $\mathrm{E}$ to detect the contamination in this section. The measured activity per unit area exceeded the Derived Working Limits (DWL) for the inactive areas, but they were less than the active areas. So, it has determined that mini-monitor $\mathrm{E}^{\prime}$ " was the most suitable for measuring lowenergy Beta -rays in ${ }^{32} \mathrm{P}$. 


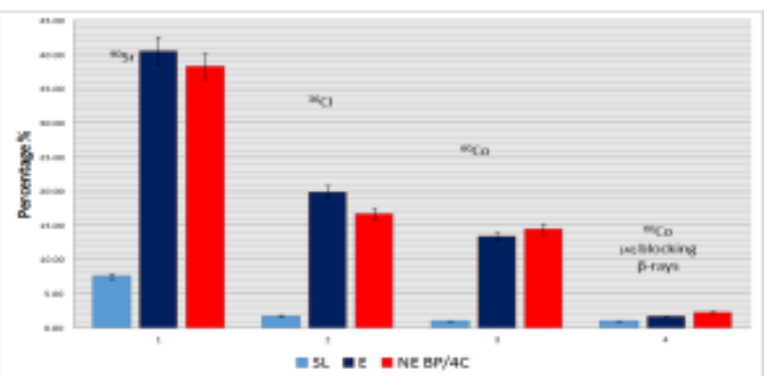

Figure 2: The efficiency of the detectors for different radiation sources.

The second part of the experiment, the contaminated surfaces were decontaminated after conducting the smear test. The G$\mathrm{M}$ tube was calibrated using the following five sources, S093.PH Sr-90, S021.RG Sr-90, S355.PH Sr-90, S354.PH Sr90 and S356.PH Sr-90. The results show that the efficiency of the G-M tube increases as the energy of the sources decreases.

Though the activity of source S355 was less than the activity of source S093 (618.73 Bq and 976.64 Bq, respectively), the efficiency of S093 was greater than the efficiency of S355 (7.4 \% and 6.1\%, respectively). See Appendix 7 in the appendices. Furthermore, the calibration of the G-M tube provided a linear relationship between the activity and the count rates of the sources as shown in Figure 3.

For the decontamination test, the wiping began at the area around the contaminated region and measuring how much this affected the activity of the contaminated area. See Appendix 8 and 9. The activity of the first wipe was $60.1 \pm 2.5$ counts/s. The following 3 wipes reduced the activity by almost $53 \%$ from the original activity level. The bar chart in Figure 4 indicates that the reduction of the rest of the wipes (a total of 13 wipes) fluctuated between $8 \%$ and $49 \%$ (see figure 4). Additionally, there was a huge change in some wipes; for example, the Wipe 8 was only half a contaminated Wipe 6 . Overall, the contamination percentage plunged dramatically throughout the decontamination process from about 60.1 counts/s to roughly 4.9 counts/s, which is just above the background level and is acceptable for ${ }^{32} \mathrm{P}$, which emits Beta rays.

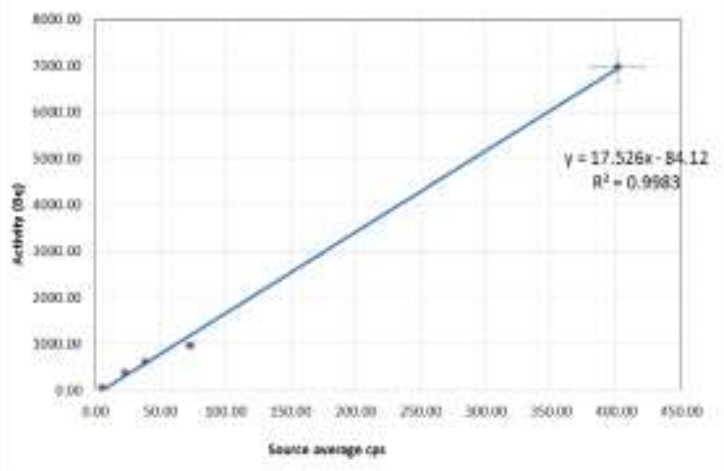

Fig. 3: Count rate vs activity of the calibration curve for the smear testing

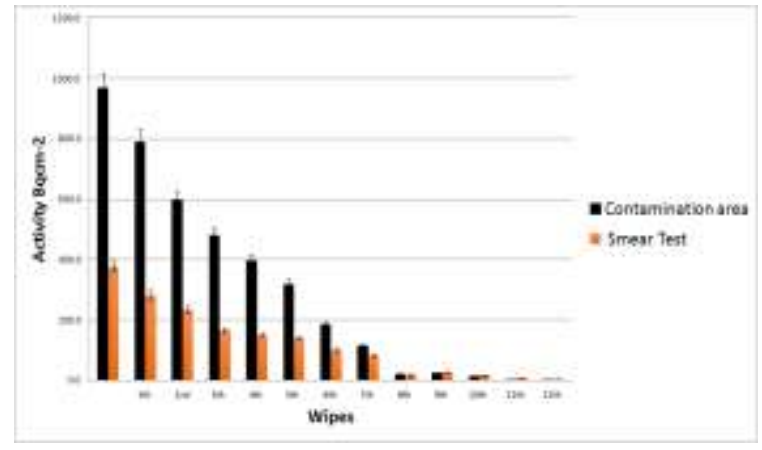

Figure 4: Reduction of the contamination by the wipes

\section{CONCLUSIONS}

The Three different detectors for surface contamination were calibrated; the result clearly shows the differences between the selected detectors for monitoring surface contamination in terms of response to the activity level and types of the radioisotopes rays. It has been found that mini-monitor $\mathrm{E}$ had a high efficiency for detecting Beta-rays. In contrast, when an aluminium sheet was used to block the low Beta-rays, the efficiency of all monitors dropped significantly. However, gamma rays were detected using NE BP4/4A with an efficiency of about $3.67 \%$. Finally, decontamination was achieved by reducing the contamination of the surface from about 60.1 counts/sec to almost 4.9 counts/s, which was just higher than the background level.

\section{ACKNOWLEDGEMENT}

All praises be to God, the most beneficent the most merciful who has made this work a successful. My profound gratitude goes to Department of Physics, University of Surrey for their support throughout this project. I would also like to thank the Department of Physics, Sokoto State University and all the hard working lecturers of the Department of Physics, Sokoto State University, for their contribution towards the success of this project.

\section{REFERENCE}

[1] Alvarez, O. B., Maia, A. F. and Caldas, L. V. E. (2005) 'Calibration procedures for hand-foot contamination monitors', Recent Advances in Multidisciplinary Applied Physics, , pp. 825\{831. doi: 10.1016/b978-008044648-6.50123-3.

[2] Baron, P. A., Kulkarni, P. and Willeke, K. (eds.) (2011) Aerosol measurement: Principles, techniques, and applications. 3rd edn. United States: Wiley, John \& Sons.

[3] Canada, G. of, Canada, H., Environments, H., Branch, C. S., Programme, S. E., Bureau, R. P., Surveillance, R. and Division, H. A. (2008) Canadian guidelines for the management of naturally occurring radioactive materials (NORM)[Health Canada, 2000]. Available at: http://www.hc-sc.gc.ca/ewh-semt/pubs/contaminants/ norm-mrn/index-eng.php (Accessed: 21 November 2015).

[4] Protection against radiation from Brachytherapy sources. Recommendations of the national council on radiation protection and measurements (1972) Annals of Internal Medicine, 77(3), p. 496. doi: 10.7326/0003-4819-77-3-496-1.

[5] Rahman, A. (2008) Decommissioning and radioactive waste management. Dun beath, Scotland, UK: Whittles Publishing. 
International Journal of Research and Scientific Innovation (IJRSI) | Volume VIII, Issue I, January 2021 | ISSN 2321-2705

\section{Appendices}

Appendix 1: Type of instrument

Instrument Type Notes

Mini-Monitor SL G-M Tube Thin metal wall $=30 \mathrm{mg} \mathrm{cm}^{-2}$ | Active length $=120 \mathrm{~mm}$ Mini-Monitor E G-M Tube Thin end window $=1.5-2.2 \mathrm{mg} \mathrm{cm}^{-2} \mid$ Area $=6.0 \mathrm{~cm}^{2}$

NE BP4/4C Scintillator Thin anthracene phosphor coupled | Sensitive area $=19.6 \mathrm{~cm}^{2}$

Appendix 2: S304.PH $\left({ }^{90} \mathrm{Sr}\right)$. Activity in $26 / 10 / 2015=3.87$ $\mathrm{kBq}$

Detector Background (cps) Count Rate $\left(\mathrm{s}^{-1}\right)$ Average Count Rate $\left(\mathrm{s}^{-1}\right)$ - (background)

Calibration Factor Efficiency \% Error $1^{\text {st }}$ Reading $2^{\text {nd }}$ Reading $3^{\text {rd }}$ Reading

Mini-Monitor "SL" 150405547.330 .557 .64 4.4, MiniMonitor IE" $0.5 \quad 706060 \quad 62.83 \quad 0.41 \quad 40.59 \quad 3.3$ and NE BP4/4A/4C 6200230170194.000 .1338 .3717 .3

Appendix 3: S358.PH $\left({ }^{60} \mathrm{Co}\right)$. Activity in $26 / 10 / 2015=1.21$ $\mathrm{kBq}$

Detector Background (cps) Count Rate $\left(\mathrm{s}^{-1}\right)$ Average Count Rate $\left(\mathrm{s}^{-1}\right)$ - (background)

Calibration Factor Efficiency \% Error 1st Reading 2nd Reading 3nd Reading

Mini-Monitor "SL" 15422.004 .041 .03 0.6, Mini-Monitor IE" 0.56786 .501 .2413 .420 .6 and NE BP4/4C 6302532 23.000 .3514 .542 .1

Appendix 4: S363.PH $\left({ }^{36} \mathrm{Cl}\right)$. Activity in 26/10/2015 $=2.7 \mathrm{kBq}$ Detector Background (cps) Count Rate $\left(\mathrm{s}^{-1}\right)$ Average Count Rate $\left(\mathrm{s}^{-1}\right)$ - (background)

Calibration Factor Efficiency \% Error 1st Reading 2nd Reading 3rd Reading

Mini-Monitor "SL" 19897.672 .35 1.77 0.3, Mini-Monitor IE" 0.520252121 .500 .8419 .911 .5 and NE BP4/4C 66070 6559.000 .3116 .722 .9

Appendix 5: S358.PH (60Co) with aluminium sheet. Activity in $26 / 10 / 2015=1.21 \mathrm{kBq}$

Detector Background (cps) Count Rate $\left(\mathrm{s}^{-1}\right)$ Average Count Rate $\left(\mathrm{s}^{-1}\right)$ - (background)

Calibration Factor Efficiency \% Error 1st Reading 2nd Reading 3nd Reading
Mini-Monitor "SL" 13242.004 .04 1.03 0.6, Mini-Monitor IE" 0.51210 .839 .691 .720 .3 and NE BP4/4C 610910 3.672 .202 .322 .9

Appendix 6: The decontamination area and Smear test measuring by using the G-M tube

No. of wipes Contaminated Area cps Error Smear Test cps Error

1th 60.12 .5526 .30 .65$, 2nd 49.90 .55211 , 3th 38.91 .5518 .2 $0.6,4$ th $32.21 .914 .30 .65,5$ th 27.41 .813 .41 .7 , 6th 22.9 2.0512 .80 .9

7 th $15.30 .8510 .60 .7,8$ th $11.41 .39 .50 .75,9$ th 61.855 .9 $0.4,10$ th 6.20 .96 .30 .2 , 11 th $5.60 .355 .70 .35,12$ th 50.55 .3 $0.45,13$ th 4.90 .750 .45

Appendix 7: Calibrating the G-M tube

Source cps Activity (Bq) Efficiency

S $356401.366979 .815 .8 \%$, S $09372.65976 .647 .4 \%$, S 355 $38.03618 .736 .1 \%$, S 021RG $22.72395 .565 .7 \%$, S 3544.71 $63.467 .4 \%$

Appendix 8: Schematic showing the surface used for decontamination experiment.

Contaminated area B, C, D, E, F, G, H and I

Appendix 9: The contamination on all the plate area. The reading (counts/second)

A 60.1, B 1.36, C 1.26, D 1.4, E 1.2, F 1.16, G 1.2, H 1.2 and I 1.23 